\title{
Synthèse du colloque
}

\section{Christine Mousny}

\section{(2) OpenEdition Journals}

Édition électronique

URL : http://journals.openedition.org/ries/2584

DOI : 10.4000/ries.2584

ISSN : 2261-4265

Éditeur

Centre international d'études pédagogiques

\section{Édition imprimée}

Date de publication : 1 mars 2000

Pagination : 101-106

ISSN : $1254-4590$

Référence électronique

Christine Mousny, « Synthèse du colloque », Revue internationale d'éducation de Sèvres [En ligne], 25 2000, mis en ligne le 01 mars 2003, consulté le 22 septembre 2020. URL : http:// journals.openedition.org/ries/2584; DOI : https://doi.org/10.4000/ries.2584 


\title{
Synthèse \\ du colloque
}

\author{
Christine Mousny \\ Responsable de programmes au CIEP
}

Les évolutions considérables survenues durant les dernières décennies $\mathrm{du} \mathrm{XX}^{\mathrm{e}}$ siècle, tant dans les domaines économiques que politiques, culturels et scientifiques, sont en passe de révolutionner en profondeur, dans le monde entier, les modes d'organisation, de pensée, de vie. Ces évolutions, parce qu'elles touchent les individus et le monde dans lequel ils vivent, auront, et ont déjà, un impact énorme sur l'éducation d'une manière générale, qu'elle soit formelle ou non formelle, à tous les niveaux. Consciente de l'ampleur des bouleversements qui s'engagent et des multiples défis que l'éducation devra relever pour les accompagner, l'UNESCO a souhaité entreprendre, dans le cadre de son Programme 1.2 pour le biennum 1998-1999, "Réforme de l'enseignement dans la perspective de l'éducation tout au long de la vie ", une réflexion sur les problèmes que posent aux États la nécessité de garantir la pleine application du droit à l'éducation, les conséquences de ce droit sur les contenus et les curricula, en envisageant la mise en place d'une réelle éducation tout au long de la vie. La Commission nationale française pour l'UNESCO s'est associée au CIEP pour répondre à ce souhait en organisant ce colloque.

En dépit des progrès accomplis au cours de ces cinquante dernières années sur l'ensemble de la planète, quelque 100 millions d'enfants n'ont toujours pas accès à l'école, 150 millions d'autres n'atteignent pas la cinquième année d'études primaires, et plus de 875 millions d'adultes sont encore analphabètes. En outre, les évolutions économiques, scientifiques, politiques, sociales et culturelles, malgré l'espoir qu'elles représentent pour l'humanité, remettent en cause l'identité des individus et leur relation au monde, et présentent un risque d'aggravation des disparités, des inégalités et des conflits. Comment, alors, offrir aux hommes et aux femmes de demain les moyens d'exister, de maîtriser leur vie, leur environnement et leur participation à la vie sociale ? Et comment s'assurer que l'éducation conservera sa vertu émancipatrice, ou qu'elle parviendra à l'acquérir ?

\section{Exiger désormais une " éducation de qualité pour tous "}

S'interroger sur le droit à l'éducation dix ans après l'engagement de Jomtien suppose d'observer les progrès accomplis, les obstacles à l'application de ce droit et les défis à relever pour prendre en compte de nouvelles attentes et de 
nouveaux besoins. Les contextes ont évolué, que ce soit aux échelles locale, nationale, régionale ou mondiale, et nécessitent de repenser les finalités de l'éducation pour qu'elles accompagnent cette évolution. Une telle réflexion porte nécessairement sur les savoirs à enseigner, les valeurs à transmettre et les méthodes à mettre en œuvre. Par conséquent, elle concerne directement les curricula. Quelle éducation ? Pour quels individus et pour quelle société ? Pour quelles valeurs ? Pour quels savoirs fondamentaux ? Pour quels savoir-faire ? Pour quels savoir-être ? Et quelle éducation fondamentale, pour quelle éducation tout au long de la vie?

Ces questions ont été à la base des réflexions des différents participants des cinq continents. Partant du constat que les dispositions internationales sur le droit à l'éducation sont "les témoins d'une volonté politique passée ", celle de la conférence de Jomtien en 1990, il a été admis qu'il est devenu désormais nécessaire de dégager " une nouvelle motivation, une nouvelle base de départ pour l'affirmation d'un nouveau droit à l'éducation, qui passe par de nouveaux contenus ", ainsi que l'a souligné Claude Durand-Prinborgne dans sa conférence introductive. L'objectif aujourd'hui ne serait plus seulement « d'obliger à faire », mais "d'obliger à faire d'une certaine manière " en visant qualité et équité, et en visant aussi des valeurs reconnues comme étant universelles telles que la paix, les droits de l'homme, la citoyenneté, tout en laissant évidemment à chaque État le soin de définir les objectifs et les finalités qui lui correspondent.

D'un objectif quantitatif (permettre que tous aient accès à l'éducation fondamentale), on en arrive à l'aube du XXI siècle à un objectif plus qualitatif. Il s'agira désormais de faire en sorte que cette éducation procure à tous les meilleures possibilités d'un développement harmonieux, d'une intégration égalitaire et équitable à leur environnement social et politique, et d'une évolution qui satisfasse leurs désirs et leurs besoins autant que ceux de la société dont ils sont membres. Il s'agira également de veiller à ce qu'elle permette à chacun d'approfondir et de conforter ses connaissances au fil de sa vie.

\section{Définir des contenus universels}

Croissance exponentielle des connaissances, des technologies, des techniques ; développement des moyens d'information et de communication ; prédominance de l'économie de marché : la liste des défis que l'éducation doit aujourd'hui relever n'est pas exhaustive. Quels qu'ils soient, ils posent tous deux problèmes majeurs : d'une part, la nécessité de traduire au niveau national des choix internationaux et, d'autre part, la difficulté à trouver le bon équilibre entre l'importance de transmettre des valeurs communes et celle de respecter les particularismes en tant qu'éléments de l'identité des individus et des groupes sociaux.

Sur la base de deux définitions des contenus - " contenus-valeurs » entendus comme ceux qui sont décidés, choisis et qui traduisent une volonté politique et sociale donnée, et « contenus-matières " qui sont la traduction péda- 
gogique, la mise en œuvre, des choix précédents -, les débats ont permis de dégager un certain nombre de points d'accord, que l'on pourrait qualifier de "réponses universelles " à l'enjeu de la recherche d'une meilleure qualité de l'éducation face aux défis qu'elle aura à relever.

En premier lieu, des contenus-valeurs ont émergé des analyses et des débats et ont été considérés comme essentiels. Unanimement reconnus comme communs et nécessaires à tous, ils visent avant tout le développement harmonieux de l'être humain. Ainsi, l'éducation devrait compter au nombre de ses missions premières celle de permettre aux enfants et aux jeunes de développer leur créativité, d'accéder à l'autonomie, d'acquérir le sens des responsabilités. En outre, elle a et aura encore, et sans doute de plus en plus, pour responsabilité de transmettre la tolérance, l'ouverture au dialogue, le respect de l'autre, et ce quels que soient les contextes géographiques, sociaux et culturels, de même qu'elle devra contribuer à la construction d'une société multiculturelle.

Ces contenus-valeurs répondent à l'idéal d'une humanité universelle, sans frontière ni race, et d'un monde plus tolérant et plus juste. Il n'est pas anodin que ces attentes resurgissent avec force en cette fin de siècle marquée par l'évanouissement des grandes idéologies, lequel a entraîné l'effacement de valeurs communes et unificatrices et l'explosion de conflits armés. L'école, et plus largement l'éducation, est alors unanimement perçue comme l'un des plus sûrs moyens d'insuffler au monde une nouvelle cohérence par les valeurs qu'elle transmet, et donc d'offrir une garantie contre l'éclatement. En ce sens, l'éducation ne saurait se limiter aux frontières des États-nations : elle a nécessairement vocation à devenir planétaire. La mondialisation est une réalité, et nier cette évidence serait courir le risque d'en subir les effets sans en avoir aucunement conscience. Il importe d'en tirer les conséquences et de fournir aux individus l'éducation qui leur permettra de ne pas subir le monde mais d'en être des acteurs conscients et solidaires.

La poursuite d'un tel idéal a également été rappelée au sujet des contenus-matières. Au cours de ce colloque, l'accent a été mis sur les sciences et les technologies. De grandes disparités sont constatées dans l'accès à ces savoirs, que ce soit entre pays développés et pays en développement ou, au sein même des pays, entre les différentes classes sociales. Et, si on n'y prend garde, elles risquent de s'aggraver. L'éducation scientifique et technologique devrait donc être partout développée et encouragée, dans un souci d'égalité d'accès à ces connaissances et à leur maîtrise.

Toutes les analyses convergent vers l'idée que l'éducation scientifique et technologique est un véritable enjeu de la citoyenneté. En effet, permettre à l'élève une véritable appropriation de ces nouveaux savoirs et savoir-faire, et notamment la maîtrise de l'ordinateur, est jugé par tous non seulement comme une condition essentielle à son insertion et à sa participation à un monde à la fois proche et lointain, mais aussi comme une ouverture vers une perception critique et constructive du progrès à l'échelle mondiale. 


\section{Prendre en compte les spécificités}

Définir des contenus universels a donc une importance majeure. Cependant, ce colloque a également été l'occasion d'une mise en garde contre le danger d'uniformisation des systèmes éducatifs dont les finalités seraient en grande partie édictées par l'ordre international. Certes, nous ne pouvons plus aujourd'hui échapper à des règles planétaires et il importe, comme cela a été évoqué ci-dessus, que l'éducation en tienne compte pour que les individus trouvent leur place dans un monde de plus en plus complexe. Néanmoins, la prise en considération de valeurs et de savoirs particuliers, liés à des situations et à des contextes précis, ne saurait être négligée : l'éducation doit pouvoir offrir à tous les moyens de comprendre leur environnement proche, de s'y insérer et d'y prendre une part active. Les participants à ce colloque ont insisté sur l'importance de contenus spécifiques adaptés aux besoins et aux attentes des populations et des sociétés.

Chaque groupe humain, par son histoire et par sa culture, est construit et se définit autour de valeurs qui lui sont propres. Bien que celles-ci puissent, à des degrés divers, rejoindre les valeurs universelles, elles se déclinent en fonction de codes et de systèmes de pensée particuliers. Remettre fondamentalement en cause ces codes et ces systèmes serait remettre en cause l'identité des civilisations 104 et des individus. C'est pourquoi l'éducation doit en tenir compte. Un exemple, longuement débattu durant le colloque, illustre bien ce problème : c'est celui du concept de démocratie, dont l'acception occidentale est difficilement comprise dans d'autres régions du monde ; ce qui engendre en conséquence des conceptions différentes de la citoyenneté. Ces divergences n'entament pas les principes universels, mais ceux-ci devraient être traduits en fonction des cadres conceptuels des sociétés. Par de tels exemples, le colloque a permis d'étayer l'idée que la définition de contenus-valeurs doit être adaptée à chaque contexte culturel, social et politique afin que l'éducation apporte véritablement à l'homme les clefs de son insertion dans la vie et dans le monde.

Par ailleurs, il est attendu des contenus de l'éducation qu'ils apportent des réponses précises à des problèmes singuliers, qui donnent lieu à une multitude de contenus-matières possibles. Qu'il s'agisse de préserver l'environnement, d'endiguer l'épidémie du sida, de contrôler les naissances, de sauvegarder les langues des minorités ethniques, les attentes envers l'éducation sont de plus en plus nombreuses.

Cette diversification des demandes qui lui sont adressées s'explique en partie par le souci des décideurs politiques de trouver des solutions positives à une série de phénomènes complexes, liés les uns aux autres, et dont les conséquences touchent l'ensemble de la structure et du fonctionnement des États, sur le plan interne comme sur le plan externe. Occuper une place satisfaisante dans 
l'économie de marché, alléger le poids de la dette extérieure, restreindre les inégalités, éviter les conflits sociaux : autant de problèmes auxquels l'éducation devrait concourir à trouver des solutions par des politiques adéquates et des formations adaptées.

Cette diversification s'explique aussi par la massification de l'accès à l'éducation et par l'élévation globale du niveau de scolarisation des populations. Mieux formés, plus informés, les individus ont une conscience plus aiguë et plus large de leurs droits, et donc de ce qu'ils sont en droit d'attendre de l'éducation. De même, ils sont plus conscients des problèmes qui se posent à la communauté à laquelle ils appartiennent, à la nation dont ils font partie. Aussi espèrent-ils de l'éducation qu'elle contribue à les résoudre par une meilleure connaissance des phénomènes.

Cette diversification s'explique enfin - et cela est lié aux deux points précédents - par une tendance de plus en plus forte à la "localisation ". Signe d'un monde qui se morcelle, qui se parcellise en sphères de pouvoir de plus en plus locales, la multiplication des pôles de décision est le pendant de la mondialisation. Le risque d'uniformisation qu'implique cette dernière engendre en réaction la recherche de systèmes plus proches et plus à l'écoute des individus, plus aptes à reconnaître, voire à mettre en valeur, les spécificités. Les instances qui interviennent dans la définition des politiques éducatives s'accroissent et augmentent du même coup l'éventail de traductions possibles des attentes diverses en contenus de l'éducation.

Du point de vue de l'éducation, la demande de prise en compte des spécificités peut être une force autant qu'une faiblesse. Son rôle s'opacifie, ses missions sont de plus en plus difficiles à cerner quand il lui est demandé de prendre en compte une multitude de facteurs, et que ces demandes émanent, qui plus est, de nombreux acteurs dont les motivations ne sont pas forcément identiques. Il est perceptible que les systèmes éducatifs auront à composer entre une multiplicité de demandes qu'il leur faudra traduire en autant de contenus, et leurs propres limites financières, institutionnelles et humaines. Ce sera sans doute l'un des défis majeurs que l'éducation aura à relever à l'avenir.

\section{Un équilibre à trouver}

La définition de nouveaux contenus de l'éducation, qu'il s'agisse des finalités ou de leur mise en ouvre pédagogique, aura à suivre corrélativement deux tendances, comme l'ont souligné les intervenants dans leurs analyses respectives. L'une a trait à des valeurs et à des savoirs universels, l'autre se rapporte à des attentes et à des besoins spécifiques. Devant ces deux lignes force, de nombreuses interrogations surgissent concernant les valeurs à inculquer, la nature et le nombre des matières à enseigner, les attitudes à développer, les méthodes à employer, les orientations à définir pour la formation des maîtres, et par consé- 
quent, les objectifs de l'éducation et les politiques à entreprendre. L'ensemble de ces interrogations peut être résumé par un questionnement : faut-il apporter aux élèves les clefs d'une intégration dans la mondialisation, ou faut-il encourager les identités culturelles fortes?

Pour les participants au colloque, les réponses restent encore à trouver. Il n'est pas question, ont-ils affirmé, d'échapper à l'une ou à l'autre voie, mais il est nécessaire de trouver le bon équilibre, la bonne mesure, selon une échelle qui reste à définir en fonction des cas. La question est de parvenir à concilier ces deux voies, l'une universelle, l'autre particulière, afin que l'éducation vise partout le même objectif de formation d'hommes et de femmes libres et responsables, mais selon des modes différents. Il s'agit de faire en sorte que ces différences dans les choix et les mises en œuvre ne soient pas sources d'inégalités ou d'affrontements destructeurs, mais qu'elles soient au contraire facteur de progrès pour tous et partout. Offrir à tous la meilleure éducation, et par conséquent répondre au double souci d'équité et de qualité, tout en accordant les moyens de comprendre et de maîtriser un environnement complexe, constitué de multiples sphères (famille, quartier, commune, région, État...), et de prendre possession de savoirs et d'instruments de plus en plus variés, telle est l'équation que les systèmes éducatifs auront à résoudre dans un proche avenir.

Ces constats, ces analyses, ces interrogations, ont pu émerger au cours de ce séminaire grâce à la grande richesse des échanges, fondés sur des expériences très diverses. Afrique, Asie, Amérique latine, Amérique du Nord, Europe : les représentants ont fait état de contextes extrêmement différents. De ce point de vue, cette réunion a permis de poser des questions essentielles, qui valent pour l'humanité aujourd'hui. Il a confirmé la nécessité d'une étape qualitative partout dans le monde et a conduit à en mesurer les difficultés. Reste maintenant nécessairement, au terme de ces réflexions, à permettre une prise de conscience générale de ces enjeux. Reste aussi, et cela est lié, à entreprendre une mobilisation de tous à tous les niveaux pour que les questions posées trouvent solution. C'est à cette ouverture sur l'avenir qu'a contribué ce séminaire : puissent ces réflexions être poursuivies. 